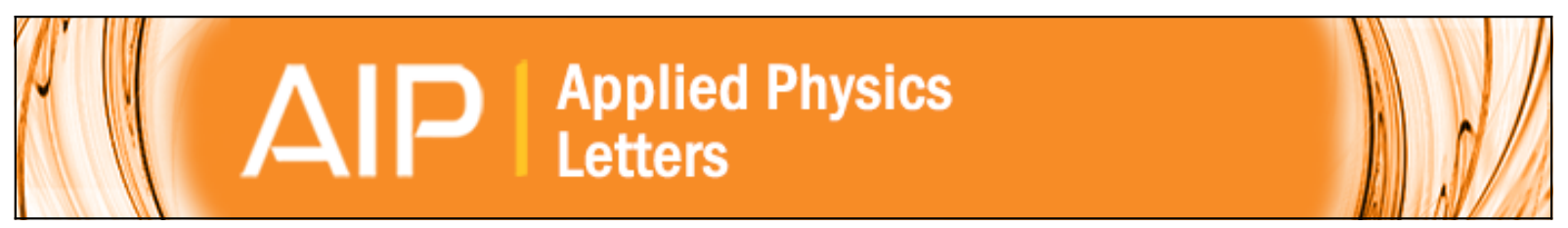

\title{
A procedure to extract the magnetocaloric parameters of the single phases from experimental data of a multiphase system
}

J. J. Ipus, L. M. Moreno-Ramírez, J. S. Blázquez, V. Franco, and A. Conde

Citation: Applied Physics Letters 105, 172405 (2014); doi: 10.1063/1.4900790

View online: http://dx.doi.org/10.1063/1.4900790

View Table of Contents: http://scitation.aip.org/content/aip/journal/apl/105/17?ver=pdfcov

Published by the AIP Publishing

\section{Articles you may be interested in}

Magnetic and magnetocaloric properties of ball milled Nd5Ge3

J. Appl. Phys. 111, 073905 (2012); 10.1063/1.3700243

Magnetocaloric effect in $\mathrm{Gd} / \mathrm{W}$ thin film heterostructures

J. Appl. Phys. 107, 09A903 (2010); 10.1063/1.3335515

Magnetization and magnetocaloric effect in ball-milled zinc ferrite powder

J. Appl. Phys. 103, 07B309 (2008); 10.1063/1.2829754

Enhanced magnetocaloric effect in single crystalline $\mathrm{Nd} 0.5 \mathrm{Sr} 0.5 \mathrm{Mn} \mathrm{O} 3$

J. Appl. Phys. 101, 09C506 (2007); 10.1063/1.2710221

The magnetocaloric effect in soft magnetic amorphous alloys

J. Appl. Phys. 101, 09 C503 (2007); 10.1063/1.2709409

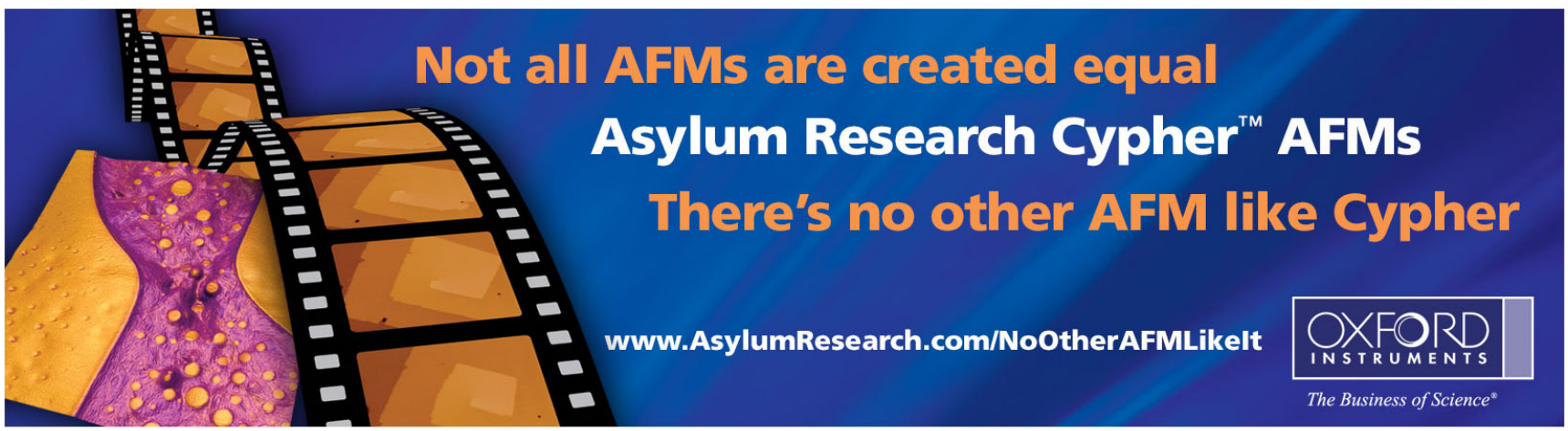




\title{
A procedure to extract the magnetocaloric parameters of the single phases from experimental data of a multiphase system
}

\author{
J. J. Ipus, L. M. Moreno-Ramírez, J. S. Blázquez, ${ }^{\text {a) }}$ V. Franco, and A. Conde \\ Departamento de Física de la Materia Condensada, ICMSE-CSIC, Universidad de Sevilla, P.O. Box 1065, \\ 41080 Sevilla, Spain
}

(Received 12 September 2014; accepted 19 October 2014; published online 29 October 2014)

\begin{abstract}
In this work, we propose a method to extract the individual parameters that describe the field dependence of magnetic entropy change of each phase in a multiphase system. This method makes use of the scaling laws of the magnetocaloric effect and can help us to determine the behavior of individual phases and to predict their critical exponents. The accuracy of the procedure is illustrated applying it to ball milled powders, in which an amorphous phase with $T_{C}$ around room temperature coexists with bcc-Fe type crystallites. The obtained values are in good agreement with those reported in the literature for single phase systems. The proposed method could be applied to other systems where single phase materials cannot be easily obtained in order to characterize the effect of impurities. (C) 2014 AIP Publishing LLC. [http://dx.doi.org/10.1063/1.4900790]
\end{abstract}

The magnetocaloric effect (MCE) and the search for magnetic materials with large MCE close to room temperature have received considerable attention in the research community for the prominent application of magnetic refrigeration close to room temperature. ${ }^{1-4}$ In fact, this environmental friendly technology is called to substitute the current gas technology.

In order to compare data obtained from different laboratories, it has to be taken into account that they can be obtained under different experimental conditions, particularly using different maximum magnetic field changes. The understanding of the field dependence of MCE has helped to get a general picture of the different magnetocaloric materials. 5,6 Moreover, the exponents defining this field dependence are related to the critical exponents of the transition, ${ }^{7}$ and there are several studies from different groups, who found a good agreement between the critical exponents extracted from conventional techniques (e.g., Kouvel-Fisher method) and the results obtained from MCE, e.g., for amorphous and nanocrystalline alloys, ${ }^{8,9}$ manganites, ${ }^{10}$ perovskites, ${ }^{11}$ and $\mathrm{Er}_{2} \mathrm{Fe}_{7}$ system. ${ }^{12}$

Most of these studies are devoted to single phase materials, although pure phases are not always easy to be obtained. Therefore, the study of the field dependence of the magnetic entropy change $\left(\Delta S_{M}\right)$ of multiphase materials is an important task. A common way to obtain $\Delta S_{M}$ is applying the Maxwell relation using the field dependence of magnetization curves as a function of temperature

$$
\Delta S_{M}=\left.\mu_{0} \int_{0}^{H_{M A X}} \frac{\partial M}{\partial T}\right|_{H} d H
$$

where $M$ is the magnetization, $H$ is the magnetic field, and $T$ is the temperature.

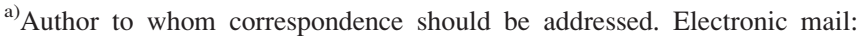
jsebas@us.es.
}

Several works have described the field dependence of $\Delta S_{M}$ following a power law, $\Delta S_{M}=a H^{n}$, where $a$ is a temperature dependent prefactor. ${ }^{13-15}$ Under this approach, there are two temperature ranges where exponent $n$ presents field independent values.

For temperatures well below the Curie temperature, $T_{C}$, and not high enough applied magnetic fields, the magnetization presents a weak field dependence. If this field dependence is temperature independent, the integral in Eq. (1) will be field independent, leading to a value of $n=1$ for this temperature range.

For temperatures well above $T_{C}$, as a consequence of the Curie-Weiss law, the magnetization has a linear dependence with the field, thus, the magnetic entropy change shows $n=2$.

At the transition temperature, for second order phase transition materials, $n$ is also field independent and, based on scaling laws, ${ }^{13}$ a value of $n=1+\frac{\beta-1}{\beta+\gamma}$ has been predicted, where $\beta$ and $\gamma$ are the critical exponents of the transition. Values of $n$ between 0.70 and 0.74 have been obtained for different amorphous and nanocrystalline alloys, 5,13,16,17 unlike $n=2 / 3$ predicted by Oesterreicher and Parker considering a mean field model. ${ }^{15}$

Therefore, relevant information about the transition can be extracted from the exponent $n$ (Ref. 7) and, in fact, MCE can be used as a tool to explore the fundamental physics in very different systems such as superconducting materials ${ }^{18}$ or graphene. ${ }^{19}$ However, several factors can yield an incorrect determination of $n$. Some of them can arise from experimental aspects, such as experimental indetermination of $T_{C}{ }^{20}$ or the use of a sample with a non negligible demagnetizing factor, $N_{D}{ }^{21,22}$ Both effects lead to artificial field dependencies of $n$ at $T_{C}$. In the former case, $n\left(T_{C}\right)$ tends asymptotically to the correct value as the magnetic field increases in the range of the experimentally available values. $^{20,23}$ In the latter case, the raw data can be corrected from the effect of the demagnetizing field if $N_{D}$ is known, ${ }^{21,22}$ using the following expression:

$$
H=H_{\text {appl }}-N_{D} M,
$$


where $H_{\text {appl }}$ is the applied magnetic field and $H$ is the internal field.

Otherwise, the knowledge of the critical behavior and the field dependence of the MCE of a particular system could lead to determine its demagnetizing factor. Besides these experimental aspects, in the case of multiphase materials with different Curie temperatures for each phase, it has been shown that, first, the exponent $n$ is field dependent and, second, it presents higher values than those showed for a single phase material at $T_{C}{ }^{24}$

In this sense, ball milling leads to microstructures that easily depart from a single phase material due to the presence of impurities or remaining untransformed phases. However, the versatility of this technique and its ability to produce metastable phases make it to be widely used to produce MCE materials. ${ }^{25-29}$ Therefore, a systematic study of the dependence of $n$ on the fraction of typical impurities found in mechanically amorphized samples, may lead to general conclusions that can be easily extended to other systems or production techniques, for which impurity phases are difficult to avoid.

We have studied in previous works the enhancement of MCE in composites (in which components have close Curie temperatures) with respect to single phase systems. ${ }^{30,31}$ In this work, we also explore multiphase systems with the aim of extracting the MCE parameters of a theoretically single phase system from the study of the field dependence of MCE in multiphase systems. In our case, the studied systems are partially amorphous $\mathrm{Fe}_{75} \mathrm{Nb}_{10} \mathrm{~B}_{15}$ alloys prepared via mechanical alloying. Details about sample preparation can be found elsewhere. ${ }^{32}$ Microstructure shows that, as milling time increases, the fraction of developed amorphous phase increases. Moreover, it was also observed that the amount of dissolved boron into the amorphous matrix increases with milling time. Isothermal magnetization data (using a LakeShore 7407 vibrating sample magnetometer) were corrected taking into account the demagnetizing factor $\left(N_{D}=1 /\right.$ 3 ), which corresponds to loosely packed spherical powders, as observed by scanning electron microscopy. ${ }^{33}$ Finally, these new curves are later used to calculate the magnetic entropy change employing Eq. (1). From the analysis of the field dependence of the magnetic entropy change presented in this study, we are able to obtain the parameters that describe the field dependence of an amorphous phase at its $T_{C}$ under the approximation of non-interacting phases.

Microstructural evolution with milling time for all studied samples can be found elsewhere. ${ }^{32}$ Milled samples show a multiphase character composed by bcc-Fe type crystals and boron inclusions embedded in an amorphous matrix. The diamagnetic character of the boron inclusions makes them irrelevant for the present study.

Figure 1(a) shows the spontaneous specific magnetization, $\sigma_{0}$, as a function of temperature. The multiphase character of the studied samples is also observed from this measurement, as $\sigma_{0}$ does not fall completely to zero above the magnetic transition temperature due to the presence of the remaining crystalline phase with a higher Curie temperature. The Curie temperature of the amorphous phase, $T_{C}{ }^{a m}$, was determined as the inflexion point in $\sigma_{0}(T)$ curves (Fig. 1(a)). Figure 1(b) shows $T_{C}{ }^{a m}$ as a function of the crystalline

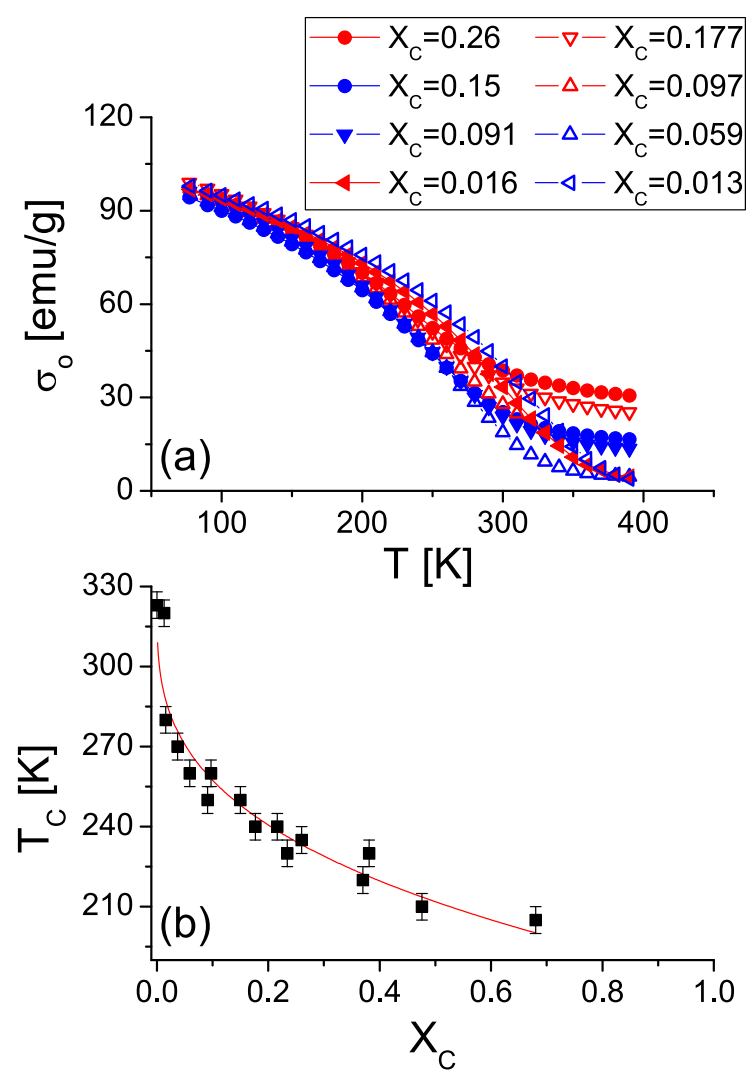

FIG. 1. (a) Spontaneous specific magnetization as a function of temperature for different crystalline fractions. (b) Curie temperature of the amorphous phase (determined as the inflexion point in magnetization curves) as a function of crystalline fraction. A rapidly quenched amorphous phase is used for $X_{C}=0$.

fraction, $X_{C}$. It can be seen that, as crystalline fraction decreases, $T_{C}{ }^{a m}$ increases, which was ascribed to higher boron content in the amorphous matrix. In fact, we have previously shown ${ }^{32}$ that boron is progressively integrated into the amorphous phase as milling time increases. Moreover, in $\mathrm{Fe}-$ based amorphous alloys, the increase in B content up to $\sim 30$ at. \% of this element leads to an increase of the Curie temperature. ${ }^{34,35}$

Figure 2(a) shows the magnetic entropy change for a maximum field change of $1 \mathrm{~T}$ as a function of temperature, for different crystalline fractions. It is observed that as crystalline fraction decreases, $\left|\Delta S_{M}\right|$ increases because the main contribution in this temperature range comes from the amorphous phase. The peak temperature also increases, in agreement with the evolution of $T_{C}{ }^{a m}$.

The temperature dependence of the exponent $n$ is shown in Figure 2(b) for several samples with different crystalline fraction. For $T \ll T_{C}{ }^{a m}$, the value of $n=1$ agrees with the expected one for materials presenting ferromagnetic behavior well below its $T_{C}{ }^{13}$ For temperatures above $T_{C}{ }^{a m}$, an increase of $n$ is observed for all curves, although the expected value for materials showing paramagnetic behavior $(n=2)^{13}$ is not reached. These values for exponent $n$ are a consequence of the biphasic character of the samples, and $n$ tends to a value between one (corresponding to ferromagnetic bcc-Fe type impurities) and two (paramagnetic amorphous phase) depending on the crystalline fraction of the sample. 


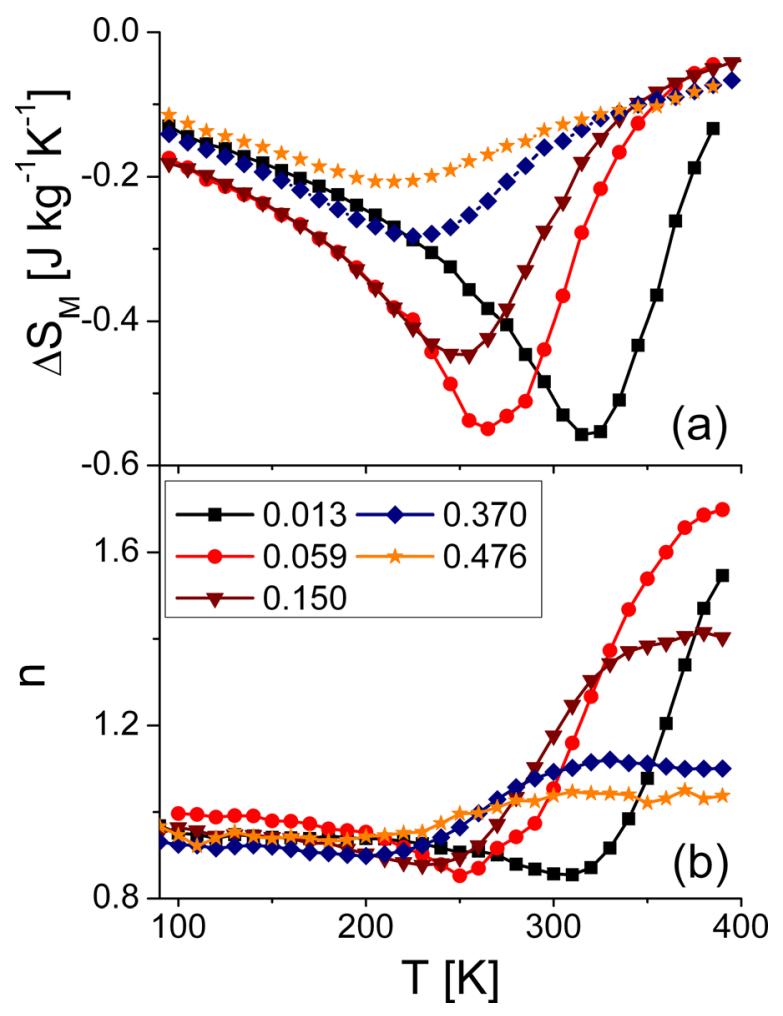

FIG. 2. Temperature dependence of (a) magnetic entropy change, $\Delta S_{M}$, and (b) field exponent, $n$, of $\Delta S_{M}$, for different crystalline fractions and a magnetic field change of $1 \mathrm{~T}$.

At the transition temperature, $n$ values are field dependent and higher than those observed for single phase amorphous alloys. ${ }^{13,36}$ As in the present case all the studied samples presents two magnetic phases, the total magnetic entropy change can be expressed as the sum of the entropy changes due to both the crystalline phase and the amorphous phase, assuming non-interacting phases

$$
\Delta S_{M}=a H^{n}=a_{C} X_{M} H^{n_{C}}+a_{A}\left(1-X_{M}\right) H^{n_{A}},
$$

where the magnetic phase fraction, $X_{M}=f X_{C}$, is related by a factor $f$ to the crystalline fraction obtained from $\mathrm{X}$-ray diffraction. ${ }^{32}$ It must be taken into account that while $X_{C}$ represents the amount of material in a crystalline array, the magnetic fraction corresponds to the amount of magnetic atoms into the crystalline phase. The prefactors $a_{C}$ and $a_{A}$ correspond to the magnetic entropy change at $1 \mathrm{~T}$ of the crystalline and amorphous phase, respectively, being temperature dependent. For the crystalline bcc-Fe type phase, the studied temperature range (around room temperature) is well below its Curie temperature, and a constant value of $a_{c}$ is a reasonable assumption because this corresponds to the low temperature tails of its expected MCE curve. For the case of the amorphous phase, changes in its $T_{C}$ with $X_{C}$, due to the dissolution of boron in the amorphous matrix were observed. Recently, it has been shown that changes in the $\mathrm{Fe} / \mathrm{B}$ ratio does not significantly affect the peak value of the entropy change for similar Fe-B based amorphous alloys, although $T_{C}$ is seriously affected. ${ }^{37}$ Therefore, the prefactor $a_{A}$ can be considered independent of $X_{C}$ at the peak temperature of each sample.
The experimental value of $n$ at the peak temperature of $\Delta S_{M}$ is related with those of each phase through the following expression:

$$
\begin{aligned}
n^{p k} & =\frac{d \ln \left(\Delta S_{M}^{p k}\right)}{d \ln (H)} \\
& =\frac{\left(X_{M} a_{C} n_{C} H^{n_{C}-1}+\left(1-X_{M}\right) a_{A} n_{A} H^{n_{A}-1}\right)}{X_{M} a_{C} H^{n_{C}}+\left(1-X_{M}\right) a_{A} H^{n_{A}}} H .
\end{aligned}
$$

Regrouping the terms,

$$
\Delta S_{M}^{p k} n^{p k}=\left(a_{C} n_{C} H^{n_{C}}-a_{A} n_{A} H^{n_{A}}\right) X_{M}+a_{A} n_{A} H^{n_{A}} .
$$

Equation (5) predicts that $\Delta S_{M}^{p k} n^{p k}$ vs $X_{C}$, for a constant $H$, should lead to a straight line where both the slope $(m)$ and the intercept $(b)$ depend on the field $H$ and are defined as

$$
\begin{gathered}
m(H)=\left(a_{C} n_{C} H^{n_{C}}-a_{A} n_{A} H^{n_{A}}\right) f, \\
b(H)=a_{A} n_{A} H^{n_{A}} .
\end{gathered}
$$

Figure 3 shows the values of $n^{p k}, \Delta S_{M}^{p k}$, and of the product $\Delta S_{M}^{p k} n^{p k}$ at $1 \mathrm{~T}$ as a function of the crystalline fraction determined by XRD. Taking logarithm to both sides of Eq. (7), the values of exponent $n_{A}$ and prefactor $a_{A}$, corresponding to the amorphous phase, could be obtained from a linear fitting of $\ln (b)$ vs $\ln (H)$ data (Fig. 4(a)), being $n_{A}=0.757 \pm 0.012$ and $a_{A}=0.596 \pm 0.011 \mathrm{~J} \mathrm{~kg}^{-1} \mathrm{~K}^{-1} \mathrm{~T}^{-0.757}$. The estimated exponent for a pure amorphous phase agrees well with typical ones

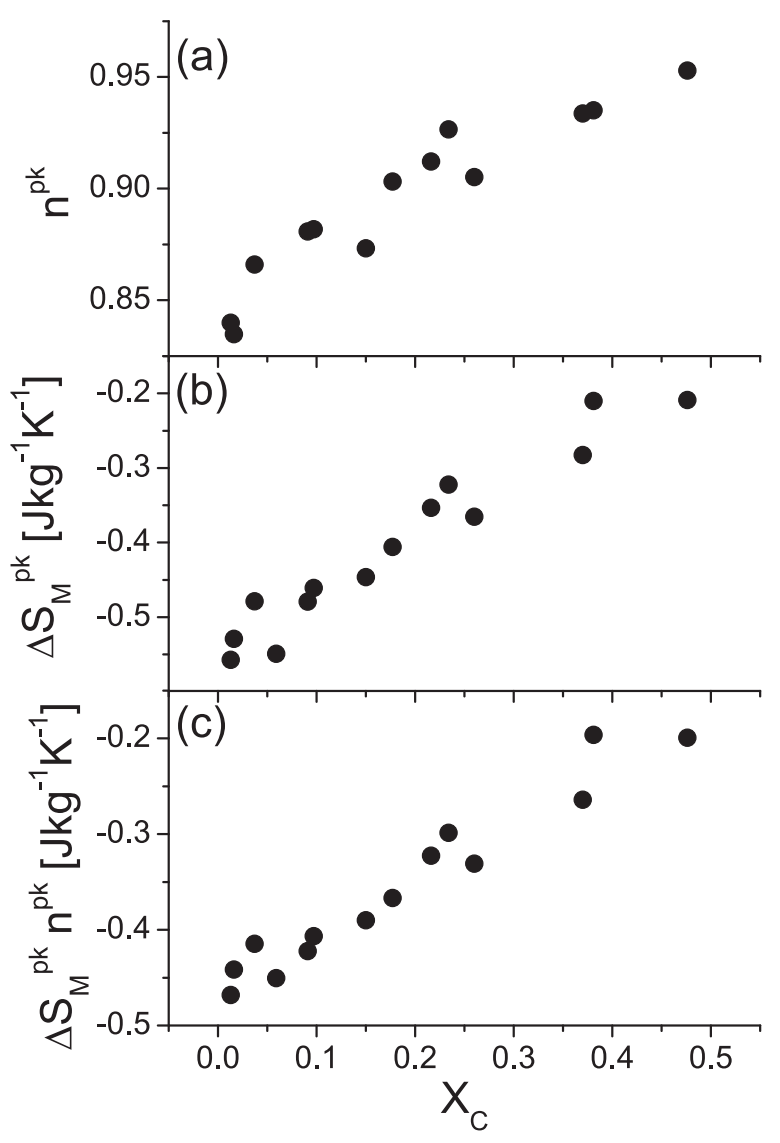

FIG. 3. Values at peak temperature of (a) $n^{p k}$, (b) $\Delta S_{M}^{p k}$, and (c) the product $\Delta S_{M}^{p k} n^{p k}$ as a function of the crystalline fraction for a magnetic field change of $1 \mathrm{~T}$. 

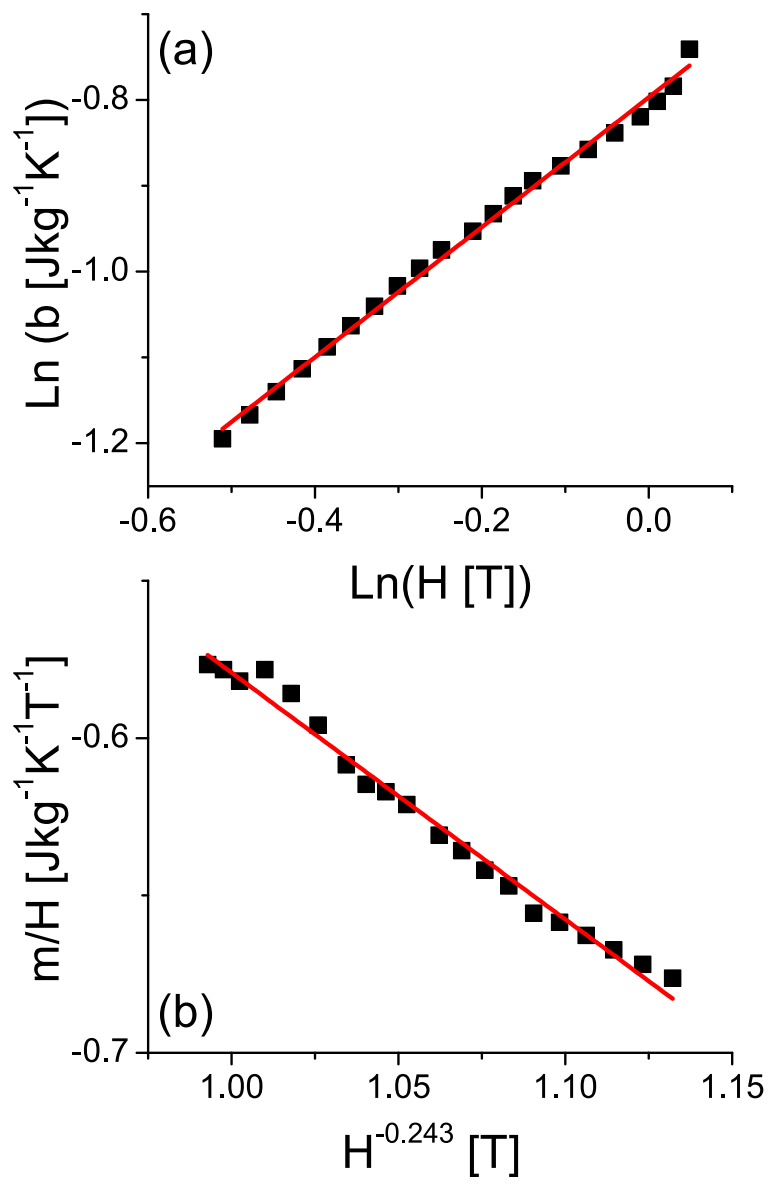

FIG. 4. Linear fitting of (a) $\ln \left(a_{A} n_{A} H^{n_{A}}\right)$ as a function of $\ln (H)$ and (b) $a_{C} f-a_{A} n_{A} f H^{n_{A}-1}$ as a function of $H^{-0.234}$. Lines correspond to the linear fit to the data.

found for other amorphous materials. ${ }^{13,34}$ The value of $a_{A}$, as expected, agrees with the limit value for $X_{C}=0$ at $\mu_{0} H=1 \mathrm{~T}$ (Fig. 3(b)).

Finally, assuming that we are well below the Curie temperature of the crystalline bcc-Fe $(\mathrm{Nb}, \mathrm{B})$ type phase, it could be assumed that $n_{c}=1$ (Ref. 7) and, therefore, Eq. (6) can be rewritten as

$$
\frac{m(H)}{H}=f a_{C}-f n_{A} a_{A} H^{n_{A}-1},
$$

where a linear fitting of $m / H$ vs $H^{n_{A}-1}$ (Fig. 4(b)) provides the values of $a_{c}=0.118 \pm 0.007 \mathrm{~J} \mathrm{~kg}^{-1} \mathrm{~K}^{-1} \mathrm{~T}^{-1}$ and $f=1.74 \pm 0.11$. The low value of $a_{c}$ is justified by the very high value of the Curie temperature of the crystalline phase. Moreover, the value of $f$ can be understood due to the higher specific magnetization of the bcc-Fe type phase $(135 \mathrm{emu} / \mathrm{g})$ with respect to that of the amorphous phase $(90 \mathrm{emu} / \mathrm{g})$, as was showed in a previous work. ${ }^{38}$

In conclusion, we have developed a model based on non interacting phases and assuming that the magnetic entropy change of a multiphase material can be expressed as the sum of the individual contributions of each phase. In the framework of this model, we have studied the field dependence of the entropy change of a composite formed by an amorphous phase, with Curie temperature close to room temperature, and remaining bcc-Fe type crystallites. The value of the experimentally obtained exponent $n$ of the composite decreases as $X_{C}$ decreases. The present analysis allows us to obtain the parameters that describe the field dependence for the amorphous phase at its $T_{C}$. The obtained results $n_{A}=0.757 \pm 0.012$ and $a_{A}=0.596 \pm 0.011 \mathrm{~J} \mathrm{~kg}^{-1} \mathrm{~K}^{-1}$ $\mathrm{T}^{-0.757}$ are in good agreement with those observed for pure amorphous materials obtained by rapid quenching. In the case of $n_{A}$, its value can be related with the critical exponents of the transition. The described method can be easily extended to analyze other systems for which pure phase materials are difficult to obtain.

This work was supported by MINECO and EU FEDER (Project MAT2013-45165-P), and the PAI of the Regional Government of Andalucía (Project P10-FQM-6462).

${ }^{1}$ K. A. Gschneidner, Jr. and V. K. Pecharsky, Annu. Rev. Mater. Sci. 30, 387 (2000).

${ }^{2}$ E. Brück, O. Tegus, D. T. C. Thanh, and K. H. J. Buschow, J. Magn. Magn. Mater. 310, 2793 (2007).

${ }^{3}$ K. A. Gschneidner, Jr. and V. A. Pecharsky, Int. J. Refrig. 31, 945 (2008).

${ }^{4}$ V. Franco, J. S. Blázquez, B. Ingale, and A. Conde, Annu. Rev. Mater. Res. 42, 305 (2012).

${ }^{5}$ V. Franco, A. Conde, V. A. Pecharsky, and K. A. Gschneidner, Jr., Europhys. Lett. 79, 47009 (2007).

${ }^{6}$ C. M. Bonilla, J. Herrero-Albillos, F. Bartolomé, L. M. García, M. ParraBorderías, and V. Franco, Phys. Rev. B 81, 224424 (2010).

${ }^{7}$ V. Franco and A. Conde, Int. J. Refrig. 33, 465 (2010).

${ }^{8}$ Z. G. Zheng, X. C. Zhong, H. Y. Yu, V. Franco, Z. W. Liu, and D. C. Zeng, J. Appl. Phys. 111, 07A922 (2012).

${ }^{9}$ V. Franco, A. Conde, V. Provenzano, and R. D. Shull, J. Magn. Magn. Mater. 322, 218 (2010).

${ }^{10}$ S. Mnefgui, N. Zaidi, A. Dhahri, E. K. Hlil, and J. Dhahri, J. Solid State Chem. 215, 193 (2014).

${ }^{11}$ T. Dang Thanh, S.-K. Oh, D.-H. Kim, S.-C. Yu, S. E. Demyanov, N. A. Kalanda, M. V. Yarmolich, and L. V. Kovalev, Curr. Appl. Phys. 14, 886 (2014).

${ }^{12}$ P. Álvarez-Alonso, P. Gorria, J. Sáncez-Marcos, J. L. SánchezLlamazares, and J. A. Blanco, J. Phys.: Condens. Matter 25, 496010 (2013).

${ }^{13}$ V. Franco, J. S. Blázquez, and A. Conde, Appl. Phys. Lett. 89, 222512 (2006).

${ }^{14}$ T. D. Shen, R. B. Schwarz, J. Y. Coulter, and J. D. Thompson, J. Appl. Phys. 91, 5240 (2002).

${ }^{15}$ H. Oesterreicher and F. T. Parker, J. Appl. Phys. 55, 4334 (1984).

${ }^{16}$ V. Franco, J. S. Blázquez, M. Millán, J. M. Borrego, C. F. Conde, and A. Conde, J. Appl. Phys. 101, 09C503 (2007).

${ }^{17}$ Q. Y. Dong, H. W. Zhang, J. R. Sun, B. G. Shen, and V. Franco, J. Appl. Phys. 103, 116101 (2008).

${ }^{18}$ N. D. Daniilidis, I. K. Dimitrov, V. F. Mitrovic, C. Elbaum, and X. S. Ling, Phys. Rev. B 75, 174519 (2007).

${ }^{19}$ M. S. Reis, Appl. Phys. Lett. 101, 222405 (2012).

${ }^{20}$ V. Franco, R. Caballero-Flores, A. Conde, K. E. Knipling, and M. A. Willard, J. Appl. Phys. 109, 07A905 (2011).

${ }^{21}$ L. M. Moreno, J. S. Blázquez, J. J. Ipus, J. M. Borrego, V. Franco, and A. Conde, J. Appl. Phys. 115, 17A302 (2014).

${ }^{22}$ C. Romero-Muñiz, J. J. Ipus, J. S. Blázquez, V. Franco, and A. Conde, Appl. Phys. Lett. 104, 252405 (2014).

${ }^{23}$ R. Caballero-Flores, V. Franco, A. Conde, and L. F. Kiss, J. Appl. Phys. 108, 073921 (2010).

${ }^{24}$ R. Caballero-Flores, V. Franco, A. Conde, Q. Y. Dong, and H. W. Zhang, J. Magn. Magn. Mater. 322, 804 (2010).

${ }^{25}$ P. Gorria, P. Álvarez, J. Sánchez-Marcos, and J. L. Sánchez-Llamazares, Acta Mater. 57, 1724 (2009).

${ }^{26}$ H. Fu, M. Zou, Q. Cao, V. K. Percharsky, K. A. Gschneidner, Jr., and L. S. Chumbley, Mater. Sci. Eng., A 528, 5219 (2011).

${ }^{27}$ P. Alvarez, S. Sánchez-Marcos, S. L. Sanchez-Llamazares, V. Franco, M. Reiffers, J. A. Blanco, and P. Gorria, Acta Phys. Pol. A 118, 867 (2010).

${ }^{28}$ F. Q. Zhao, W. Dagula, O. Tegus, T. J. Gortenmulder, E. Bruck, and K. H. J. Buschow, IEEE Trans. Magn. 41, 3754 (2005).

${ }^{29}$ Y. X. Zhang, Z. G. Liu, H. H. Zhang, and X. N. Xu, Mater. Lett. 45, 91 (2000). 
${ }^{30}$ R. Caballero-Flores, V. Franco, A. Conde, K. E. Knipling, and M. A. Willard, Appl. Phys. Lett. 98, 102505 (2011).

${ }^{31}$ S. C. Paticopoulos, R. Caballero-Flores, V. Franco, J. S. Blázquez, A. Conde, K. E. Knipling, and M. A. Willard, Solid State Commun. 152, 1590 (2012).

${ }^{32}$ J. J. Ipus, J. S. Blázquez, C. F. Conde, J. M. Borrego, V. Franco, S. Lozano-Pérez, and A. Conde, Intermetallics 49, 98 (2014).

${ }^{33}$ J. J. Ipus, J. S. Blázquez, V. Franco, and A. Conde, Intermetallics 16, 1073 (2008).
${ }^{34}$ C. L. Chien and K. M. Unruh, Phys. Rev. B 25, 5790 (1982).

${ }^{35}$ I. Kaban, P. Jovari, A. Waske, M. Stoica, J. Bednarcik, B. Beuneu, N. Mattern, and J. Eckert, J. Alloys Compd. 586, S189 (2014).

${ }^{36}$ R. Caballero-Flores, V. Franco, A. Conde, K. E. Knipling, and M. A. Willard, Appl. Phys. Lett. 96, 182506 (2010).

${ }^{37}$ V. Franco, C. F. Conde, J. S. Blázquez, A. Conde, P. Svec, D. Janickovic, and L. F. Kiss, J. Appl. Phys. 101, 093903 (2007).

${ }^{38}$ J. J. Ipus, J. S. Blázquez, V. Franco, A. Conde, and L. F. Kiss, J. Appl. Phys. 105, 123922 (2009). 